Lipoprotein lipase is a key enzyme in lipid metabolism, ensuring the hydrolysis of plasma triglycerides found in chylomicrons and very low density lipoproteins. An increase in the catalytic activity of lipoprotein lipase leads to a decrease in the level of TG and an increase in the level of $\mathrm{HDL}$ in the blood. According to the results of the survey in children with JIA, the concentration of lipoprotein lipase was $13.9[7.1 ; 25.3]$ $\mu \mathrm{g} / \mathrm{l}$, with obesity $27.5[9.9 ; 47.8] \mu \mathrm{g} / \mathrm{l}$. A direct relationship was established between its content in blood serum and the concentration of LDL ( $r s=0.37 ; p<0.05)$ and the inverse with the level of $\operatorname{LL}$ ( $r s=-0.4$; $p<0.05)$. There was a significant difference $(p<0.05)$ by gender: the level of lipoprotein lipase in the blood serum in girls was 25.8 [16.7 - 54.4] $\mu \mathrm{g} / \mathrm{l}$, in boys - 15.6 [7.8 - 29,0] $\mu \mathrm{g} / \mathrm{l}$. A correlation was also established between the level of lipoprotein lipase and body mass index ( $r s=0.35$; $\mathrm{p}<0.05)$.

According to the results of the study, a significant $(p<0.05)$ increase in the level of primary (DK233) and secondary (DK278 and MDA) LPO products in the blood serum of children with JIA was found in comparison with the control group. During the correlation analysis, a positive correlation was established between the levels of DK233, DK278 in serum and ESR ( $r s=0.587, p<0.01$ ). The results obtained indicate the intensification of lipid peroxidation processes. A significant $(p<0.05-0.01)$ decrease in the content of water-soluble (ACW) and fat-soluble (ACL) antioxidant capacity of substances in the blood serum of children with JIA was found when compared with similar indicators in the control group. During the correlation analysis, a negative correlation was established between the level of CRP and the content of ACL in serum ( $r$ s = -0.346, $p<0.05$ ), between the content of ACW and CRP in serum ( $r$ s = $0.54, p<0,01)$.

Conclusion: It can be concluded that the activation of lipid peroxidation and the failure of the antioxidant system play a significant role in the development and progression of JIA in the examined children, as well as in the formation of atherogenic disorders by the lipid peroxidation mechanism. In addition, children with JIA have abnormalities in the lipid spectrum, acquiring an atherogenic orientation, which, together with hemostasiological changes, can be regarded as a risk factor for atherothrombosis.

Disclosure of Interests: None declared

DOI: 10.1136/annrheumdis-2019-eular.7633

\section{SAT0492 GASTROINTESTINAL MANIFESTATIONS IN CHILDHOOD BEHÇET'S DISEASE}

Ferhat Demir ${ }^{1}$, Nelgin Gerenli ${ }^{2}$, Seval Simsek ${ }^{1}$, Betül Sözeri ${ }^{1}{ }^{1}$ University of Health Sciences, Umraniye Training and Research Hospital, Pediatric Rheumatology, Istanbul, Turkey, ${ }^{2}$ University of Health Sciences, Umraniye Training and Research Hospital, Pediatric Gastroenterology, Istanbul, Turkey

Background: Behçet's disease (BD) is an idiopathic, chronic, inflammatory systemic vasculitis characterized by recurrent oral and genital aphthous ulcers, skin lesions, and ocular involvement. It can also affect multiple visceral organ and system such as heart, lung, blood vessels, gastrointestinal (GI) system, and central nervous system (1). The etiology of the disease is still unknown. New diagnostic criteria for pediatric Behçet's patients have been established In 2015, and its largely based on clinical findings (2). Although the Gl manifestations are not take part in to the diagnostic criteria, it can be emerge in great deal of patients and associated with significant morbidity and mortality. The most common Gl symptoms in BD are abdominal pain, nausea, diarrhea and Gl bleeding in, respectively. Also, the most common reported site of intestinal involvement was ileocecal. The literature data related to $\mathrm{Gl}$ involvement of BD are frequently concern adult patients and pediatric studies are limited. Objectives: We analyzed the Gl manifestations and its endoscopic findings in children diagnosed with BD.

Methods: We retrospectively reviewed the clinical records of 64 patients who fulfilled the international pediatric classification criteria for BD from December 2015 to December 2018. Endoscopic examination results of 13 patients were also collected and analyzed

Results: Of the 64 children with BD, 37 were male and 27 were female, and sex ratio (male/female) was 1.37/1. The mean age of our patients was $9.49 \pm 4.10$ and $11.52 \pm 3.50$; at onset of symptoms and at the time of diagnosis, respectively. Assessment of our 64 patients revealed that the prevalence of $\mathrm{Gl}$ symptoms is $60 \%(39 / 64)$. The most frequent Gl symptoms were abdominal pain in $56 \%$ (36/64), chronic diarrhea in $18 \%$ (12/ $64)$, nausea/vomiting in $11 \%(7 / 64)$, chronic constipation in $12 \%(8 / 64)$, and regurgitation or heartburn in 7\% (5/64). Upper Gl endoscopy was performed in 7 patients and colonoscopy in 6 . All of the patients who underwent the endoscopy or colonoscopy, had findings support the $\mathrm{Gl}$ inflammation. The endoscopic findings shown in the patients are; gastric inflammation in $100 \%(7 / 7)$, oesophageal inflammation in $57 \%(4 / 7)$, duodenal inflammation in $42 \%(3 / 7)$, colonic erythema and inflammation in $83 \%(5 / 6)$, polyps in $50 \%(3 / 6)$, ulceration in $66 \%(4 / 6)$, and hemorrhoids in $16 \%(1 / 6)$. Upper Gl ulceration was not observed. Pathology specimens were taken in 7 upper endoscopies and 6 colonoscopies within our patients. The findings of pathology specimens were vascular congestion and inflammation in $100 \%(7 / 7)$ of upper Gl biopsies and $83 \%(5 / 6)$ of colonic biopsies, cryptitis in $66 \%(4 / 6)$ of colonic biopsies. Acute colitis in pathology specimens was shown in $83 \%(5 / 6)$ of the patients. Three of the patients with colitis had punch ulcers, and these patients were evaluated as BD-associated colitis. The other two patients were diagnosed and treated as Crohn's colitis accompanying BD.

Conclusion: The Gl system is one of the most frequently affected organ by the $\mathrm{BD}$. It may cause the inflammation and ulceration in the $\mathrm{Gl}$ tract Inflammatory bowel diseases can also cause Gl inflammation similar to BD. In patients with symptoms of $\mathrm{Gl}$ inflammation, upper and lower $\mathrm{Gl}$ endoscopic examination and pathological analysis may be helpful in determine the underlying etiology.

\section{REFERENCES}

[1] - Sakane T, Takeno M, Suzuki N, Inaba G. Behçet's disease. N Engl J Med 1999;341:1284-91

[2] - Koné-Paut I, Shahram F, Darce-Bello M, Cantarini L, Cimaz R, Gattorno $M$ et al, PEDBD group. Consensus classification criteria for paediatric Behçet's disease from a prospective observational cohort: PEDBD. Ann Rheum Dis. 2016;75:958-64.

Acknowledgement: We are grateful to all participating children and their families.

Disclosure of Interest: None Declared

DOI: 10.1136/annrheumdis-2019-eular.4053

\section{SAT0493 THE CHALLENGE OF TREATING PULMONARY VASCULITIS IN BEHÇET'S DISEASE: TWO PEDIATRIC CASES}

Selcan Demir ${ }^{1}$, Erdal Sag ${ }^{1}$, Ummusen Kaya Akca $^{1}$, Tuncay Hazirolan ${ }^{2}$ Yelda Bilginer', Seza Özen'. '1 Hacettepe University Faculty of Medicine, Pediatric Rheumatology, Ankara, Turkey, ${ }^{2}$ Hacettepe University Faculty of Medicine, Radiology, Ankara, Turkey

Background: Behçet's Disease (BD) is a multisystemic autoinflammatory disease and the most severe complication of BD is pulmonary artery involvement (PAI). Data regarding treatment and outcomes of pediatric patients with PAI is very limited.

Objectives: Herein, we report two pediatric patients with BD presenting with PAI and treated successfully with aggressive immunosuppressive treatment.

Methods: Demographic data, clinical manifestations, laboratory and radiological findings, and treatments of patients were documented from patient charts retrospectively.

\section{Results:}

Case 1: A 15-year-old boy was admitted with abdominal pain and fever. An abdominal Doppler ultrasonography (USG) showed stenosis of vena cava inferior $(\mathrm{VCl})$ with a thrombus. Transthoracic echocardiography (TTE) detected that the thrombus extended from $\mathrm{VCl}$ to the right atrium. When he started to have hemoptysis, he was referred to our hospital. TTE showed a mass in RV. The computed chest and abdominal tomography angiography (CTA) showed bilateral aneurysmatic dilatation with thrombi in the pulmonary arteries and thrombosis in vena hepatica. The pathergy test was negative and the HLA B5 was negative. According to the ICBD the patient had been diagnosed as BD due to genital ulcers and vascular involvement. He was given pulse methylprednisolone (MP) $500 \mathrm{mg}$ days along and followed by oral prednisolone $1 \mathrm{mg} / \mathrm{kg} / \mathrm{day}$, intravenous cyclophosphamide at a dose of $15 \mathrm{mg} / \mathrm{kg}$ every 3 weeks for a total of 6 cycles, and Interferon- $\alpha 2 a$ (IFN- $\alpha 2 a) 3$ times a week. Within one month, hemoptysis and fever disappeared, and CRP values normalized. After a three-month treatment, TTE and CTA revealed that thrombi shrank significantly. The dosage of prednisolone was tapered gradually and stopped 2 years later. Immunosuppressive treatment was continued with adalimumab. The patient has been followed in remission for nearly 6 years.

Case 2: A fifteen-year-old boy was referred to our hospital for the evaluation of fever for over 4 months and a thrombus in his right ventricle. $\mathrm{He}$ had a medical history with cough, fever, intermittent hemoptysis and weight loss for the past 3 months. Physical examination revealed acnelike rashes over face and back, oral ulcers. A CTA confirmed the 
thrombus in RV and showed bilateral multiple aneurysms along the pulmonary artery and its branches. According to ICBD, the patient was diagnosed with $\mathrm{BD}$ due to having aphthous ulcers, pseudofolliculitis, and vascular involvement. Iv MP (500 mg/day) for 3 days was followed by oral prednisolone $1 \mathrm{mg} / \mathrm{kg} / \mathrm{day}$, which was subsequently tapered. Iv cyclophosphamide at a dose of $500 \mathrm{mg}$ was also given every 3 weeks for a total of 6 cycles, followed by oral azathioprine (AZA). Concomitant subcutaneous IFN- $\alpha 2$ a was given two times per week for 6 months. Within two weeks, cough and fever disappeared, CRP values normalized. After 1 year the pulmonary artery aneurysm disappeared and cardiac thrombosis resolved. We have been following the patient with AZA for four years without recurrence.

Conclusion: We present two pediatric patients with pulmonary involvement of $\mathrm{BD}$. PAI is a life-threatening condition and should be managed with more aggressive medical therapy. Early diagnosis and aggressive immunosuppressive treatment are very important in PAl. We strengthened our treatment with IFN- $\alpha 2 a$. There is no data in the literature regarding the use of IFN- $\alpha-2 a$ in PAl treatment along with low dose cyclophosphamide. There were no mortality or recurrences within the 6 and 4 years follow up period. An aggressive immunosuppressive therapy leads to better prognosis in this most dreadful complication of BD.

\section{REFERENCES}

[1] Ozen S, Bilginer Y, Besbas N, Ayaz NA, Bakkaloglu A. Behcet disease: treatment of vascular involvement in children. Eur J Pediatr. 2010;169 (4):427-30.

Disclosure of Interests: Selcan Demir: None declared, Erdal Sag: None declared, Ummusen Kaya Akca: None declared, Tuncay Hazirolan: None declared, Yelda Bilginer: None declared, Seza Özen Consultant for: Seza Ozen is receiving consultancy fees from Novartis, Speakers bureau: Roche

DOI: 10.1136/annrheumdis-2019-eular.4946

\section{SAT0494 HOME MONITORING OF INACTIVE DISEASE IN CHILDREN WITH JUVENILE IDIOPATHIC ARTHRITIS PREDICTIVE VALUE OF EQ-5D-5L-Y}

Martijn J.H. Doeleman ${ }^{1}$, Sytze De Roock ${ }^{1}$, Nathan Buijsse ${ }^{1}$, Mark Klein ${ }^{1}$, Gouke J. Bonsel ${ }^{2}$, V. Seyfert ${ }^{3}$, Nico Wulffraat ${ }^{1}$, Joost F. Swart ${ }^{1}$. ${ }^{1}$ University Medical Center Utrecht, Pediatric Rheumatology, Utrecht, Netherlands; ${ }^{2}$ University Medical Center Utrecht, Obstetrics and Gynaecology, Utrecht, Netherlands; ${ }^{3}$ MyOwnMed, Bethesda, United States of America

Background: In recent years, juvenile idiopathic arthritis (JIA) research has shifted towards treat-to-target therapy based on clinical assessments and patient-reported outcomes (1). A well-known measurement of quality of life is the EQ-5D-5L (2). Herewith, we report preliminary results of a retrospective study using the child-friendly ' $E Q-5 D-5 L-Y$ ' with an E-health application (Reuma2GO) to monitor children with JIA in an outpatient setting.

Objectives: To assess the relationship between dimensions of the healthrelated quality of life 'EQ-5D-5L-Y' questionnaire and conventional assessments for children with JIA, including the Juvenile Arthritis Multidimensional Assessment Report (JAMAR) and active joint count (AJC), and to investigate the potential of the EQ-5D-5L-Y as instrument for outpatient management.

Methods: The study was designed as monocentric retrospective cohort study. Data from October 2017 to January 2019 were available for 70 patients with JIA. The relationships between individual dimensions of the EQ-5D-5L-Y, JAMAR and several clinical assessments were investigated. Furthermore, dimensions of the EQ-5D-5L-Y were investigated as possible predictors for binary disease activity using AJC $>0$ as reference standard for active disease.

Results: Seventy patients with JIA completed 115 EQ-5D-5L-Y and JAMAR questionnaires within two weeks before a clinical visit. Moderate to high correlations were found between the EQ-5D-5L-Y and JAMAR. Moreover, the best possible EQ-5D-5L-Y score, with and without healthrelated visual analogue scale (EQ-VAS), demonstrated high sensitivity $(81.1 \%)$ and negative predictive value $(84.8 \%)$ for active disease (Table 1). The few patients who were incorrectly classified as having inactive disease (false-negatives) did not have their medication changed at the clinical visit and experienced little to no impact of disease activity on their quality of life, as indicated by the JAMAR questionnaire.
Table 1. Predictive value of EQ-5D-5L-Y dimensions for active disease using AJC $>0$ as reference standard.

\begin{tabular}{|c|c|c|c|c|c|}
\hline Cut-off for 'Active Disease' & $\begin{array}{c}\text { Accuracy }(\%, \\
\text { Cl) }\end{array}$ & Sensitivity & Specificity & PPV & NPV \\
\hline EQ-5D Mobility > 1 & $\begin{array}{c}74.8 \%(65.8- \\
82.4)\end{array}$ & $59.5 \%$ & $82.1 \%$ & $61.1 \%$ & $81.0 \%$ \\
\hline EQ-5D Self-Care $>1$ & $\begin{array}{c}76.5 \%(67.7- \\
83.9)\end{array}$ & $35.1 \%$ & $96.2 \%$ & $81.2 \%$ & $75.8 \%$ \\
\hline EQ-5D Usual Activities > 1 & $\begin{array}{c}67.8 \%(58.5- \\
76.2)\end{array}$ & $73.0 \%$ & $65.4 \%$ & $50.0 \%$ & $83.6 \%$ \\
\hline EQ-5D Pain/Discomfort > 1 & $\begin{array}{c}62.6 \%(53.1- \\
71.5)\end{array}$ & $62.2 \%$ & $62.8 \%$ & $44.2 \%$ & $77.8 \%$ \\
\hline $\begin{array}{l}\text { EQ-5D Anxiety/Depression } \\
>1\end{array}$ & $\begin{array}{c}73.9 \%(64.9- \\
81.7)\end{array}$ & $51.4 \%$ & $84.6 \%$ & $61.3 \%$ & $78.6 \%$ \\
\hline EQ-5D EQ-VAS <85 & $\begin{array}{c}60.0 \%(50.4- \\
69.0)\end{array}$ & $64.9 \%$ & $57.7 \%$ & $42.1 \%$ & $77.6 \%$ \\
\hline EQ-5D Total Score $>5$ & $\begin{array}{c}60.0 \%(50.4- \\
69.0)\end{array}$ & $81.1 \%$ & $50.0 \%$ & $43.5 \%$ & $84.8 \%$ \\
\hline $\begin{array}{l}\text { EQ-5D Score }>5 \text { \& EQ- } \\
\text { VAS }<85\end{array}$ & $\begin{array}{c}52.2 \%(42.7- \\
61.6)\end{array}$ & $83.8 \%$ & $37.2 \%$ & $38.8 \%$ & $82.9 \%$ \\
\hline
\end{tabular}

PPV: positive predictive value; NPV: negative predictive value.

Conclusion: These results demonstrate the discriminatory value of the EQ-5D-5L-Y between active and inactive disease in our cohort of patients with JIA. High negative predictive value was found for the total EQ-5D$5 \mathrm{~L}-\mathrm{Y}$ score, with and without EQ-VAS. In conclusion, the EQ-5D-5L-Y could be a valuable instrument for monitoring children with JIA in an outpatient setting which could aid physicians with deciding whether a clinical visit is necessary or not.

\section{REFERENCES}

[1] Filocamo G, et al. A new approach to clinical care of juvenile idiopathic arthritis: The juvenile arthritis multidimensional assessment report. J Rheumatol 2011;38:938-953.

[2] Janssen MF, et al. Measurement properties of the EQ-5D-5L compared to the EQ-5D-3L across eight patient groups: a multi-country study. Qual Life Res 2013;22:1717-1727.

Disclosure of Interests: Martijn J.H. Doeleman: None declared, Sytze De Roock: None declared, Nathan Buijsse: None declared, Mark Klein: None declared, Gouke J. Bonsel: None declared, V. Seyfert Shareholder of: VS is CEO and founder at MyOwnMed, Inc., Employee of: VS is CEO and founder at MyOwnMed, Inc., Nico Wulffraat: None declared, Joost F. Swart: None declared

DOI: 10.1136/annrheumdis-2019-eular.4509

\section{SAT0495 PHENOTYPE OF PATIENTS WITH JUVENILE DERMATOMYOSITIS ASSOCIATED WITH ANTI-FACTOR H AUTO-ANTIBODY : A SEVERITY FACTOR?}

Cécile Dumaine $^{1}$, Isabelle Melki ${ }^{1}$, Brigitte Bader-Meunier ${ }^{2}$, Cyril Gitiaux ${ }^{2}$, MarieAgnes Durey ${ }^{3}$. ${ }^{1}$ Hôpital Robert-Debré Ap-Hp, Paris, France; ${ }^{2}$ Hôpital Necker, Paris, France; ${ }^{3}$ Hospital European Georges-Pompidou AP-HP, Paris, France

Background: Juvenile Dermatomyositis (JDM) is a rare, autoimmune and highly heterogeneous paediatric-onset myopathy, characterized by skin and muscles inflammation. Development of vasculopathy is associated with the severe extra-muscular manifestations of JDM, and portends a poor prognosis. Impaired function of JDM vasculature includes immune complex and complement deposition.

Objectives: The aim of our study is to describe the clinical and paraclinical phenotype of patients with anti-factor $\mathrm{H}$ autoantibody (Ab) associated with JDM and overlap myositis.

Methods: Patients with a diagnosis of JDM or overlap myositis and presence of anti-FH auto-antibody, followed in two Parisian tertiary centers over the last twelve years, were retrospectively selected. Besides demographic data, clinical features (cutaneous lesions, severity of muscle involvement, extra-muscular manifestations), treatment options and their outcome were retrieved for each patient. Biological data and presence of myositis-specific autoantibodies were collected, muscle MRI were examined, and data from muscle biopsies were assessed using a validated score tool when available.

Results: Eleven female patients were included in the study, with a median age at diagnosis of 8.8 years [2.8 - 13.1], and a median follow- 